\title{
Pacientes com Hipovitaminose D Podem Não Ter Hiperparatireoidismo Secundário
}

\section{carta ao editor}

\section{ROBERTA VANACOR \\ Melissa Orlandin Premaor tania Weber Furlanetto}

Universidade Federal do Rio Grande do Sul (RV); Serviço de Medicina Interna do Hospital de

Clínicas de Porto Alegre (MOP, TWF); Porto Alegre, RS, Brasil s resultados apresentados no artigo escrito por Silva e cols. (1) são relevantes para a prática médica, pois mostram hipovitaminose $\mathrm{D}$ (HVD) em uma área tropical do Brasil (paralelo $19^{\circ}$ ) e estabelecem um ponto de corte para esse diagnóstico, medindo $25(\mathrm{OH}) \mathrm{D}$ no soro por HPLC. No entanto, observa-se que neste estudo, similarmente a estudos realizados pelo presente grupo e por outros, há um subgrupo de indivíduos com deficiência de vitamina $\mathrm{D}$ que não tem hiperparatireoidismo secundário (HPS). Este achado apresenta importância clínica, pois o grupo com HVD e sem HPS parece comportar-se de maneira diferente do grupo com HPS. Chen e cols. (2) observaram que o HPS em indivíduos com deficiência de vitamina $\mathrm{D}$ se associou ao aumento de mortalidade. Adicionalmente, no presente estudo foi observado aumento do número de internações hospitalares nos indivíduos com deficiência de vitamina D e HPS, quando comparados aos com deficiência de vitamina sem HPS (3).

Várias causas têm sido relacionadas à presença ou não de HPS na hipovitaminose $\mathrm{D}$. Uma delas é a deficiência de magnésio, que causaria hipoparatireoidismo funcional (4). Esse achado foi verificado recentemente em adultos jovens (5). Indivíduos com menor ingestão de cálcio também seriam mais propensos a apresentar HPS, quando deficientes de vitamina D (6). Um estudo realizado pelo presente grupo de pesquisa em pacientes com deficiência de vitamina $\mathrm{D}$, internados em hospital geral, mostrou maior aumento do hormônio da paratireóide em indivíduos normoalbuminêmicos, quando comparados aos hipoalbuminêmicos, sugerindo papel da ligação às proteínas na biodisponibilidade da $25(\mathrm{OH}) \mathrm{D}(7)$. Outros fatores também podem estar associados à presença ou não de HPS na HVD, por exemplo, fatores genéticos (8), índice de massa corporal $(9,10)$ e uso de diuréticos tiazídicos $(11,12)$.

Provavelmente, a causa da ausência de HPS na HVD é multifatorial, no entanto, mais estudos devem ser realizados para melhor esclarecimento das causas e implicações deste fenômeno.

As autoras são patrocinadas pelo Fundo de Incentivo à Pesquisa do Hospital de Clínicas de Porto Alegre (Fipe) e pelo Ministério da Educação (Capes) por meio do Programa de Pós-graduação em Medicina: Ciências Médicas da Universidade Federal do Rio Grande do Sul (UFRGS). 


\section{REFERÊNCIAS}

1. Silva BCC, Camargos BM, Fujii JB, Dias EP, Soares MMS. Prevalência de deficiência e insuficiência de vitamina $D$ e sua correlação com PTH, marcadores de remodelação óssea e densidade mineral óssea, em pacientes ambulatoriais. Arq Bras Endocrinol Metabol. 2008;52(3):482-7.

2. Chen JS, Sambrook PN, March L, Cameron ID, Cumming RG, Simpson JM, et al. Hypovitaminosis D and parathyroid hormone response in the elderly: effects on bone turnover and mortality. Clin Endocrinol (Oxf). 2008;14;68(2):290-8.

3. Premaor MO, Scalco R, da Silva MJS, Furlanetto TW. Secondary hyperparathyroidism is associated with increased risk of hospitalization or death in elderly adults living in a geriatric institution. Gerontology [In press].

4. Sahota O, Mundey MK, San P, Godber IM, Hosking DJ. Vitamin D insufficiency and the blunted PTH response in established osteoporosis: the role of magnesium deficiency. Osteoporos Int. 2006;17(7):1013-21.

5. Premaor MO, Paludo P, Manica D, Paludo AP, Rossatto ER, Scalco R, et al. Hypovitaminosis D and secondary hyperparathyroidism in resident physicians of a general hospital in southern Brazil. J Endocrinol Invest. 2008. In press.

6. Steingrimsdottir L, Gunnarsson O, Indridason OS, Franzson L, Sigurdsson G. Relationship between serum parathyroid hormone levels, vitamin $D$ sufficiency, and calcium intake. JAMA. 2005;9;294(18):2336-41.

7. Premaor MO, Alves GV, Crossetti LB, Furlanetto TW. Hyperparathyroidism secondary to hypovitaminosis $D$ in hypoalbuminemic is less intense than in normoalbuminemic patients: a prevalence study in medical inpatients in southern Brazil. Endocrine. 2004;24(1):47-53.
8. Binkley N, Novotny R, Krueger D, Kawahara T, Daida YG, Lensmeyer $G$, et al. Low vitamin $D$ status despite abundant sun exposure. J Clin Endocrinol Metab. 2007;92(6):2130-5.

9. Kamycheva E, Sundsfjord J, Jorde R. Serum parathyroid hormone level is associated with body mass index. The $5^{\text {th }}$ Tromso study. Eur J Endocrinol. 2004;151:167-72.

10. Premaor MO, Paludo P, Manica D, Paludo AP, Rossatto ER, Scalco $R$, et al. Hypovitaminosis $D$ and secondary hyperparathyroidism in resident physicians of a general hospital in southern Brazil. J Endocrinol Invest. 2008 [In press].

11. Scalco R, Premaor MO, Fröehlich PE, Furlanetto TW. High prevalence of hypovitaminosis $D$ and secondary hyperparathyroidism in elders living in nonprofit homes in South Brazil. Endocrine. 2008;33(1):95-100.

12. Rejnmark $L$, Vestergaard $P$, Heickendorff $L$, Andreasen $F$, Mosekilde L. Effects of thiazide- and loop-diuretics, alone or in combination, on calcitropic hormones and biochemical bone markers: a randomized controlled study. J Intern Med. 2001;250(2):144-53.

Endereço para correspondência:

Melissa Premaor

Serviço de Medicina Interna do Hosp. das Clínicas de Porto Alegre.

Rua Ramiro Barcelos, 2350

90035-003 Porto Alegre RS

E-mail:mopremaor@bol.com.br 